\title{
Erratum to: Highly efficient and stable white LEDs based on pure red narrow bandwidth emission triangular carbon quantum dots for wide-color gamut backlight displays
}

Fanglong Yuan ${ }^{1, \S}$, Ping $\mathrm{He}^{1, \S}$, Zifan $\mathrm{Xi}^{1, \S}$, Xiaohong $\mathrm{Li}^{1}$, Yunchao $\mathrm{Li}^{1}$, Haizheng Zhong ${ }^{2}$, Louzhen Fan ${ }^{1}(\bowtie)$, and Shihe Yang $^{3}(\Delta)$

${ }^{1}$ College of Chemistry, Beijing Normal University, Beijing, 100875, China

${ }^{2}$ Beijing Key Laboratory of Nanophotonics and Ultrafine Optoelectronic Systems, School of Materials Science and Engineering Beijing Institute of Technology, Beijing, 100081, China

${ }^{3}$ Guangdong Key Lab of Nano-Micro Material Research, School of Chemical Biology and Biotechnology, Shenzhen Graduate School, Peking University, Shenzhen, 518055, China

${ }^{\S}$ Fanglong Yuan, Ping He, and Zifan Xi contributed equally to this work.

(C) Tsinghua University Press and Springer-Verlag GmbH Germany, part of Springer Nature 2020

\section{Erratum to}

Nano Research 2019, 12(7): 1669-1674

https://doi.org/10.1007/s12274-019-2420-x

In the version of this article originally published, Figs. 2(d) and 3(a), and Figs. S3 and S6 in the Electronic Supplementary Material (ESM) were incorrect. The corrected images are shown below.
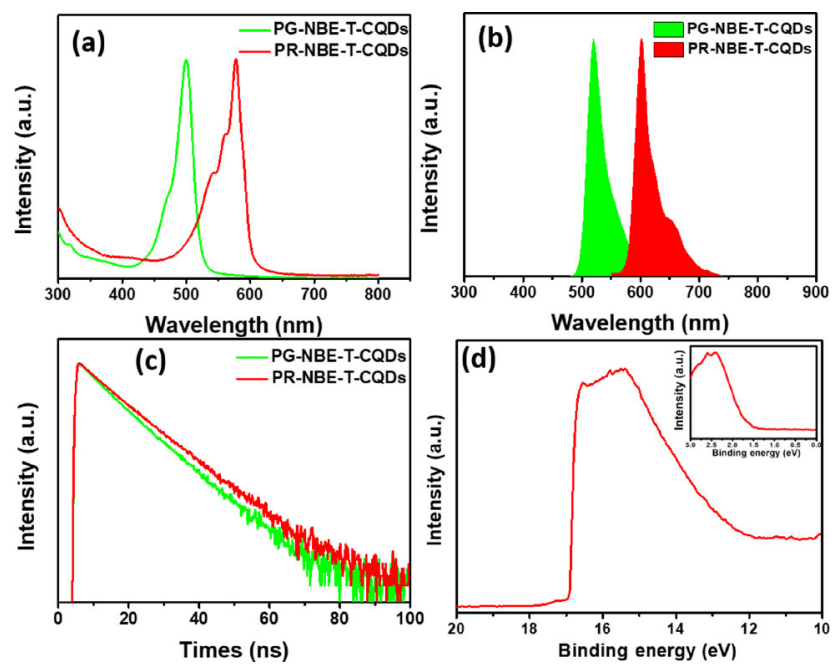

Figure 2 (a) UV-vis absorption spectra, (b) PL emission spectra (excited at $365 \mathrm{~nm}$ ) and (c) time-resolved PL decay profiles of PR- and PG-NBE-T-CQDs. (d) UPS of PR-NBE-T-CQDs (inset shows the Fermi level region).
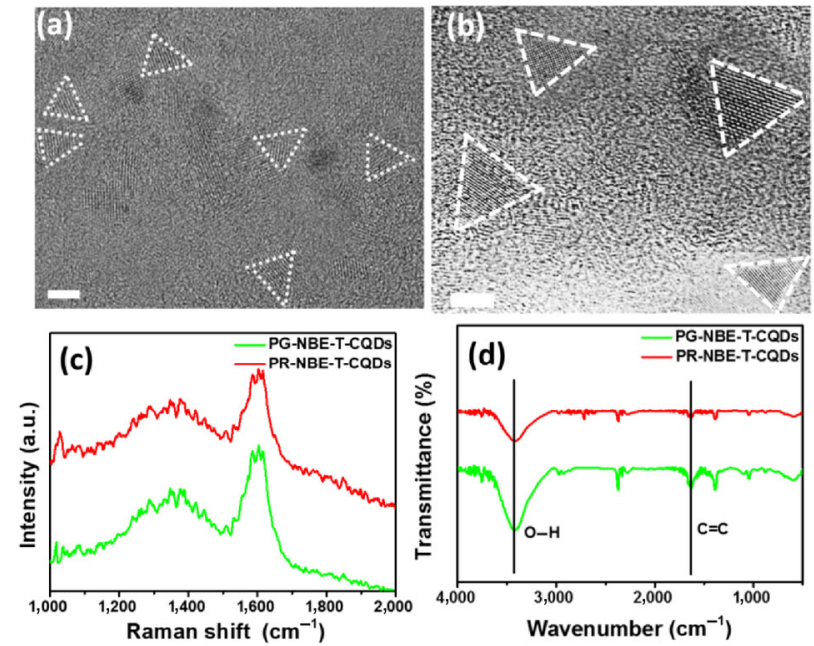

(e)

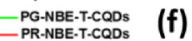

PG-NBE-T-CQDS
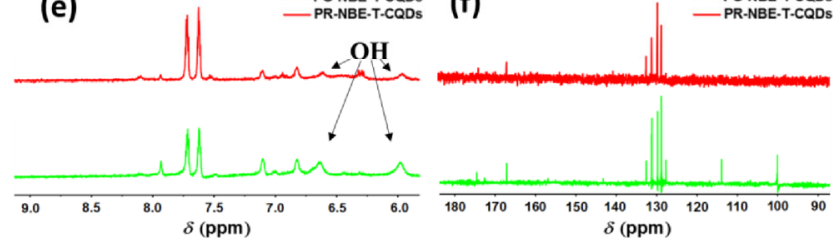

Figure 3 The wide-area TEM images of (a) PG- and (b) PR-NBE-T-CQDs, the scale bar is $2 \mathrm{~nm}$. (c) Raman spectra of PR- and PG-NBE-T-CQDs. (d) FT-IR spectra of PR- and PG-NBE-T-CQDs. (e) ${ }^{1} \mathrm{H}$ NMR spectra and $(f){ }^{13} \mathrm{C}$ NMR spectra of PR- and PG-NBE-T-CQDs. 


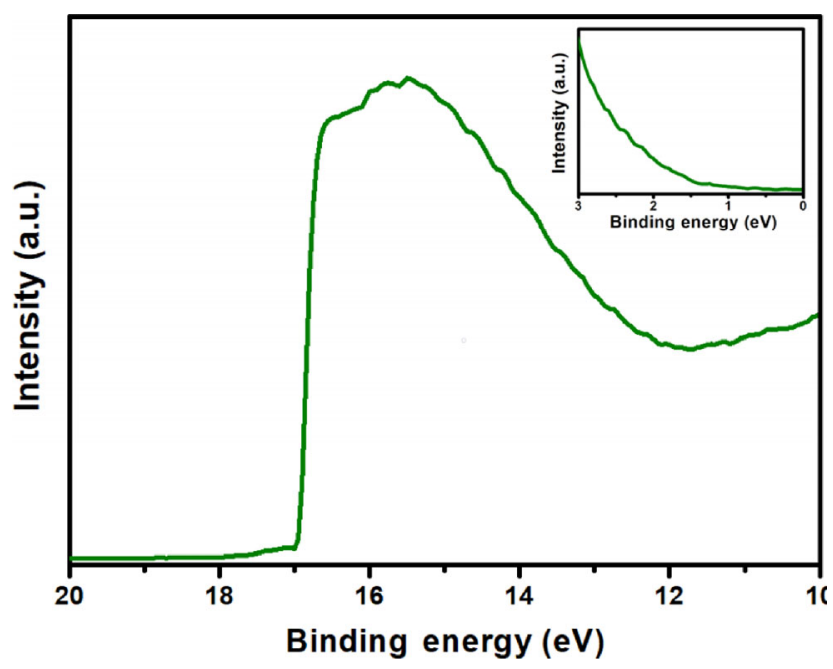

Figure S3 Ultraviolet photoelectron spectroscopy (UPS) data of PGNBE-T-CQDs.

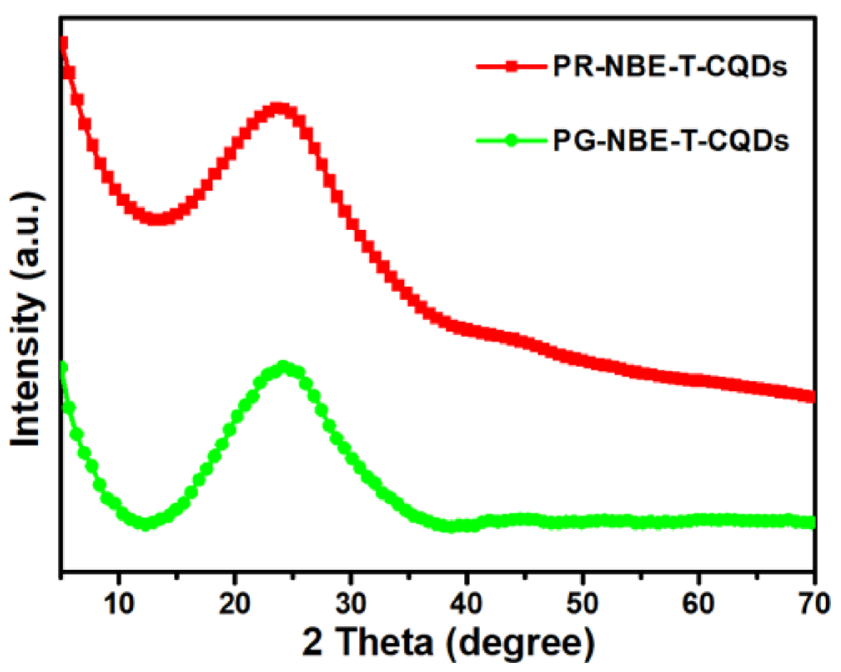

Figure S6 XRD pattern of PG- and PR-NBE-T-CQDs.

The online version of the original article can be found at https://doi.org/10.1007/s12274-019-2420-x 